\title{
Importance and Limitations of Modeling Parasitic Capacitance between Package and PCB for Power Bus Noise and Radiation
}

\author{
Umberto PAOLETTI $^{\dagger \text { a) }}$, Takashi HISAKADO ${ }^{\dagger}$, and Osami WADA ${ }^{\dagger}$, Members
}

SUMMARY Power and ground planes on multilayer PCBs can effectively radiate electromagnetic fields excited by the IC simultaneous switching noise. The high frequency electromagnetic radiation is often calculated from the electric field along the edge of the PCB, which can be estimated with a cavity model using magnetic walls. The excitation of the cavity modes is related to the via current passing through the power bus planes at the interconnection between IC package and PCB. Usually the attention is focused on the differential-mode current of the package pins, but in the present paper it is shown that the common-mode current flowing out from package pins plays a very important role in the excitation of cavity modes, and its neglect implies a fatal underestimation of the electromagnetic radiation from the power bus planes in some circumstances. A second important contribute to the radiation is given by the common mode current on the pins, together with the current flowing on the PCB ground plane. With the proposed equivalent circuit, the effectiveness of decoupling inductors depending on their location and on the value of the parasitic capacitance is studied.

key words: common mode, parasitic capacitance, package, simultaneous switching noise

\section{Introduction}

It is well known that the high frequency switching of digital circuits generates noise on the power supply system, and that this conducted noise can become an effective source of radiated emissions at PCB level, where the dimensions can be comparable with the wavelength. In particular, the power and ground planes of multilayer PCBs can radiate very effectively already at few hundreds MHz. This effect can be efficiently modeled for example by means of a LECCS model (e.g. in [1]) representing the IC and driving the resonances of the power/ground planes (e.g. in [2]), which can be calculated with a cavity model using vertical magnetic walls (e.g. in [3]).

However, when the frequency increases, the effect of the package and its interaction with the PCB cannot be neglected, and even the radiation will be affected. In order to verify this effect, an interesting experiment has been reported in [4]. The DUT consists of a small module supplied by a battery on a two layer PCB. On the power and supply lines at module level, two inductors have been placed, $L_{g d i}$ and $L_{v d i}$, representing either the package parasitic inductances, or two decoupling inductors. In order to scale the problem to lower frequencies, where the IC can be more

\footnotetext{
Manuscript received September 16, 2008.

${ }^{\dagger}$ The authors are with the Department of Electrical Engineering of the Graduate School of Kyoto University, Kyoto-shi, 6158510 Japan.

a)E-mail: paoletti@ circuit.kuee.kyoto-u.ac.jp

DOI: 10.1587/transcom.E92.B.1937
}

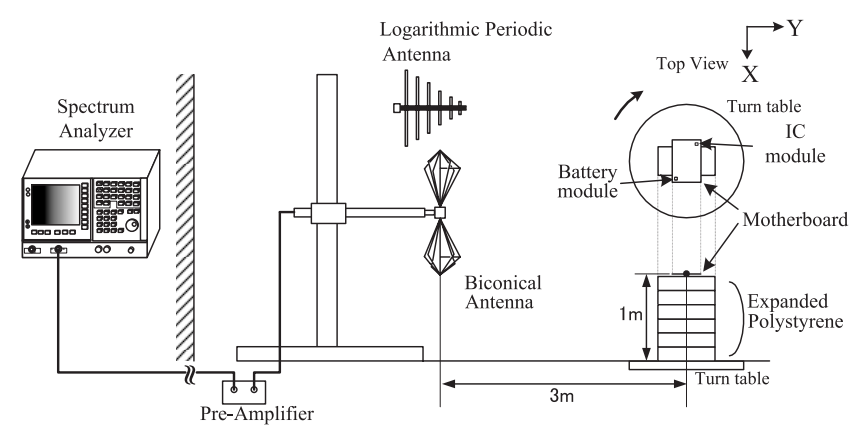

Fig. 1 Measurement setup for radiated emissions [4], [5] and [6].

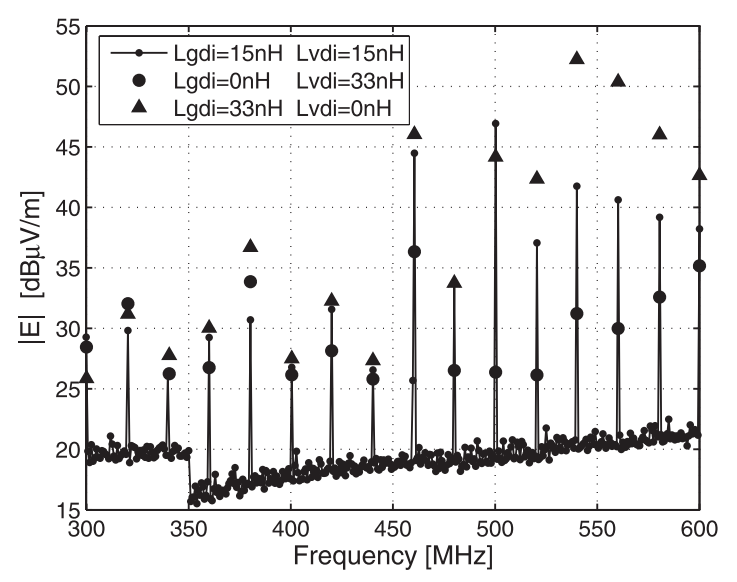

Fig. 2 Measured electric field [4]-[6] and [7].

easily modeled, relatively large inductance values have been selected. The vertical component of the radiated electric field has been measured in a semi-anechoic chamber with the measurement setup shown in Fig. 1.

Three different configurations of inductors have been used, and the maximum measured electric field for any azimuth is shown in Fig. 2 for the frequencies between 300 and $600 \mathrm{MHz}$. It is very interesting to observe that even though the sum of the inductance values is almost the same, there is a very large difference up to $20 \mathrm{~dB}$ in the radiation above $450 \mathrm{MHz}$ when the inductors are placed either on the power or on the ground line. This cannot be explained with a simple cascade connection of the models of IC, module, inductors and power/ground planes, as it is often done in package modeling, because only the total loop inductance would be significant.

A thorough study of the module, which is partially de- 
scribed in [8], showed that a very small parasitic capacitance $(0.85 \mathrm{pF})$ bypasses the inductor on the ground line, and causes considerable differences already below $1 \mathrm{GHz}$, but it cannot explain the differences at frequencies below $600 \mathrm{MHz}$.

In the present work an alternative solution for this problem will be proposed. It will be shown that a common mode current at package level, which is generated by a differential source at IC level, can strongly affect the current on the via in the power/ground planes of the PCB, and ultimately affect the radiation related to the resonances of the power/ground planes. Furthermore, the common mode on the pins combined with the antenna-type current on the PCB ground plane are not completely negligible at some of the considered frequencies.

The fundamental role played by the parasitic capacitance between PCB ground plane and module ground plane will be shown. Some limitations related to the use of this parasitic capacitance in the equivalent circuit will be also discussed.

In Sect. 2 the DUT will be shortly described. In Sect. 3 the equivalent circuit will be presented. The considered radiation mechanisms will be discussed in Sect. 4. The estimated via and common mode current will be presented in Sect. 5, together with the calculated radiated field. Finally, the results will be summarized in Sect. 6 .

\section{Description of the DUT}

For the purpose of the present work, only a rough description of the DUT is necessary. Further details can be found for example in [4] and [8]. The DUT is a four layer module of dimensions $25 \mathrm{~mm} \times 25 \mathrm{~mm}$. The thickness of the FR4 substrate is $0.28 \mathrm{~mm}-1.0 \mathrm{~mm}-0.28 \mathrm{~mm}(t=1.6 \mathrm{~mm})$. On layers 2 and 4 two ground planes are present, and on layer 3 a power plane, as shown schematically in Fig. 3. The two ground planes are connected through an assembly of ground vias on the periphery of the module. The parasitic capacitance between the power and ground planes has been estimated to be $C_{p g}=25 \mathrm{pF}$, based on measurements and simulations, as shown in [8].

On layer 1 a CMOS oscillator, which is made with one of the six inverters of the IC (74LVC04), drives the parallel connection of the remaining five. The clock frequency is $20 \mathrm{MHz}$. Very close to the IC on the module, a decoupling capacitor is present on the power supply system $\left(C_{b}=1 \mathrm{nF}\right.$, $E S L=1 \mathrm{nH}$, and the resistance $E S R=0.1 \Omega$ had no effect), with a $2.8 \mathrm{nH}$ parasitic interconnect inductance. The crystal and the other components have not been shown in Fig. 3.

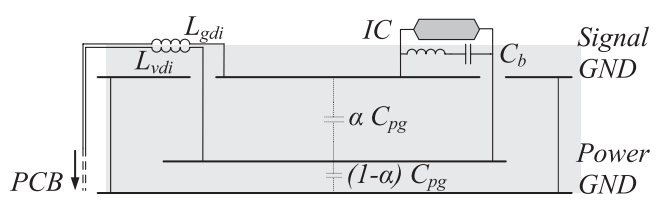

Fig. 3 Simplified representation of the DUT [5] and [6].
The module is supplied by a battery module on a two-layer $1.6 \mathrm{~mm}$ thick PCB, which simply consists of a pair of rectangular power/ground planes of dimensions $235 \mathrm{~mm} \times 320 \mathrm{~mm}$. IC and battery modules are mounted on opposite corners of the PCB, as shown in Fig. 1.

The connection between the IC module and the PCB consists of two thin vertical pins, which are connected to vias on the module from the bottom layer to the top one, as schematically represented in Fig. 4. The distance between the PCB and the module has been recorded to be about $3 \mathrm{~mm}$, but unfortunately the DUT is not available anymore, and the exact distance could not be verified again. In the present work this value is attributed to the distance between the top ground plane of the PCB and the top ground plane of the module $(t+h)$.

On the top layer of the module the power supply vias are connected to two parallel traces of $6.8 \mathrm{~mm}$ length, $0.2 \mathrm{~mm}$ width, and $0.2 \mathrm{~mm}$ separation from each other. At the end of the power and ground traces two inductors are placed, $L_{v d i}$ and $L_{q d i}$, respectively. Immediately after the inductors, the ground trace is connected to the ground plane on layer 2 , and the power trace is connected through a via to the power plane on layer 3 .

\section{Equivalent Circuit Model}

The equivalent circuit for the DUT mounted on the PCB is shown in Fig. 5. For the IC the LECCS model $\left(I_{I C}, Z_{I C}\right)$ calculated in [4] has been used. The bypass capacitor, $C_{b}$, the discrete inductors on the power and ground lines, $L_{v d i}$ and $L_{g d i}$, and the power/ground capacitance on the module, $C_{p g}$, appear explicitly in the circuit.

The remaining parasitic elements on the module have been calculated with FASTCAP [9] and FASTHENRY [10], and in part have been confirmed by the measurement results shown in [8]. All the parameters of the module have been calculated without considering the PCB in the simulations. In particular, the parasitic capacitance between the ground line on the module and the module ground plane, that is the sum $C_{1}+C_{2}$, has been estimated to be $0.85 \mathrm{pF}$.

The inductances $L_{v p i n}$ and $L_{g p i n}$ represent the partial in-

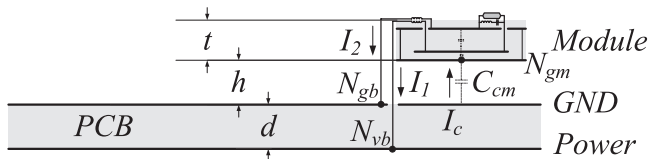

Fig. 4 Connection of the DUT to the PCB [6].

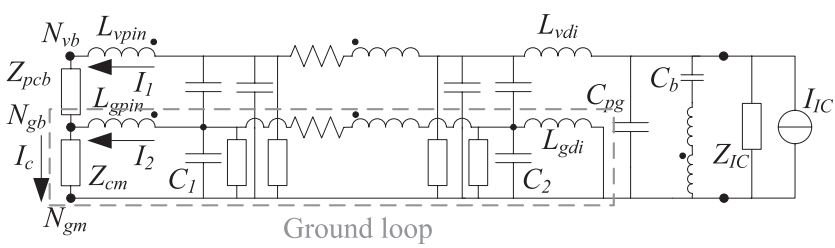

Fig. 5 Lumped element equivalent circuit with complex impedance [7]. 


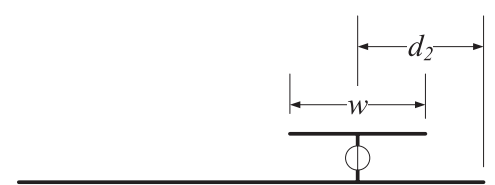

(a)

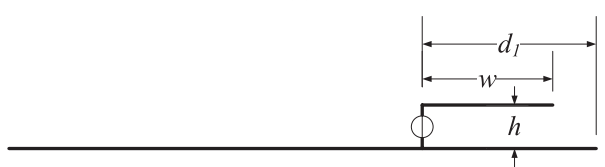

(b)

Fig. 6 Front (a) and side (b) view of PCB and module in the MoM model.

ductances of the pins connecting the module to the power and ground planes, respectively. Their estimated values were $2.9 \mathrm{nH}$ and $1.9 \mathrm{nH}$, with a mutual inductance of $1.1 \mathrm{nH}$. For the input impedance of the power/ground planes on the PCB, $Z_{p c b}$, the cavity model described in [3] has been used.

In the schematic the module ground plane $N_{g m}$ has been used as reference, and not the PCB ground plane $N_{g b}$. The parasitic impedance $Z_{c m}$ between module ground, $N_{g m}$, and PCB ground, $N_{g b}$, can be represented with a capacitance at low frequencies. The value of the capacitance is strictly related to the distance between module ground and PCB ground, which unfortunately is not known exactly, as already mentioned. Its value has been estimated with FASTCAP to be $2.8 \mathrm{pF}, 3.8 \mathrm{pF}$ and $5 \mathrm{pF}$ for distances $h$ between the two ground planes of $3 \mathrm{~mm}, 2 \mathrm{~mm}$ and $1.4 \mathrm{~mm}$, respectively. Last value corresponds to a $3 \mathrm{~mm}$ distance between the top ground plane of the PCB and the top layer of the module $(t+h)$, and has been used in the rest of this work.

As the frequency increases, the parasitic capacitance alone is not enough to model the parasitic impedance. The complex impedance $Z_{c m}$, between module ground, $N_{g m}$, and PCB ground, $N_{g b}$, has been calculated with a full wave method of moment (MoM) tool [11] (EMSURF).

The approximate physical positions of the two ground nodes are shown in Fig. 4 . The node $N_{g b}$ corresponds to the connection point of the pin to the PCB ground plane and its position can be clearly determined. However, the exact position of the node $N_{g m}$ on the small module ground plane is not clear. In the MoM calculations its position has been set at the edge of the module ground, exactly above the node $N_{g b}$.

The front and side views of the simplified model are shown in Fig. 6. The model consists of two conducting planes connected to an ideal voltage generator. The width $w$ corresponds to the $25 \mathrm{~mm}$ width of the square module. The height $h$ was $1.4 \mathrm{~mm}$, and the distances $d_{1}$ and $d_{2}$ were $40 \mathrm{~mm}$ and $25 \mathrm{~mm}$, respectively.

The calculated impedance $Z_{c m}$ is shown in Figs. 7 and 8. In the same figures also the impedance of the $5 \mathrm{pF}$ capacitance value is shown, which corresponds to the capacitance between two ground planes at $1.4 \mathrm{~mm}$ distance calculated with FASTCAP. In order to write an equivalent circuit, the complex impedance has been fitted with a series RLC cir-

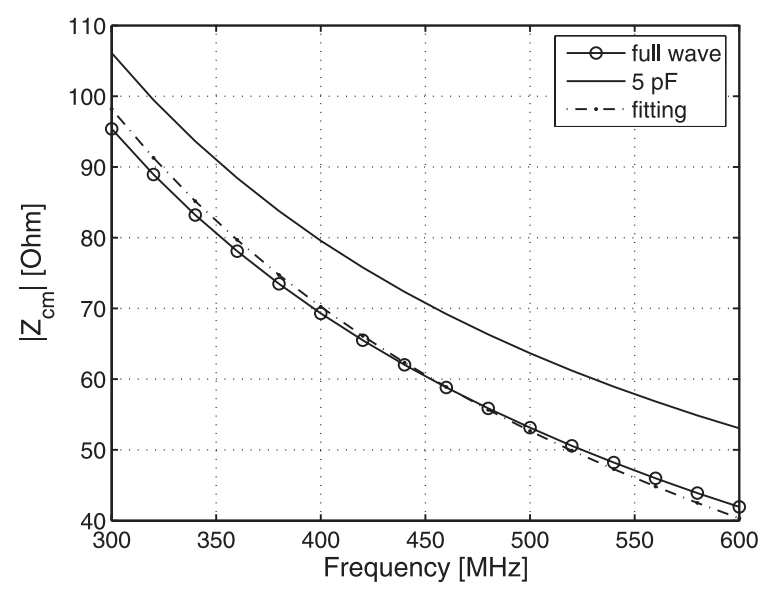

Fig. 7 Magnitude of estimated impedance $Z_{c m}$.

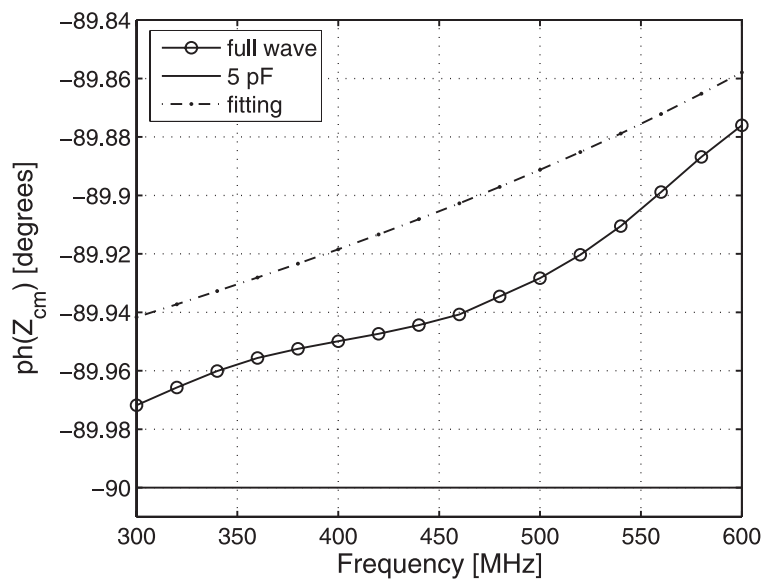

Fig. 8 Phase of estimated impedance $Z_{c m}$.

cuit in the frequency range between $300 \mathrm{MHz}$ and $600 \mathrm{MHz}$ $\left(R_{c m}=0.1 \mathrm{Ohm}, C_{c m}=5.1 \mathrm{pF}, L_{c m}=3.1 \mathrm{nH}\right)$.

\section{Calculation of the Radiated Field}

The PCB was in horizontal position in the semi-anechoic room as shown in Fig. 1, at $1.5 \mathrm{~m}$ height for the frequencies below $350 \mathrm{MHz}$, and at $1 \mathrm{~m}$ height for the frequencies above $350 \mathrm{MHz}$. During measurement the PCB was rotated and the maximum value at each frequency of the vertical polarization ( $z$-direction) of the electric field at $3 \mathrm{~m}$ distance was recorded.

The calculation has been split into several separated stages by considering two radiation mechanisms. For each radiation mechanism a complex transfer function between a unit current and the complex electric field in one angular position at $3 \mathrm{~m}$ distance has been calculated with the methods described in the following subsections. In both cases, the contribute to the electric field due the floor of the semianechoic room has been considered by applying the method of images. 


\subsection{Radiation from the Power/Ground Planes}

The first excitation mechanism of the radiation is the via current in the PCB power/ground planes, $I_{1}$, and is related to the resonances of the parallel plates. By assuming a unitary current in the via of the PCB power/ground planes, it is possible to calculate the voltage along the edge of the PCB power/ground planes using the equation for the transfer impedance $Z_{i j}$ obtained with the cavity model in [3].

From the voltage $V$ along the edge of the PCB and the thickness $d$ of the board, the electric field along the edge can be calculated $(V=E d)$. A simple and rough estimation of the radiated electromagnetic field can be obtained by using equivalent surface magnetic current sources $\mathbf{M}_{\mathbf{s}}$ along the edge:

$$
\mathbf{M}_{\mathbf{s}}=-\hat{\mathbf{n}} \times \mathbf{E}
$$

where $\hat{\mathbf{n}}$ is the normal to the lateral surface of the power/ground planes, and it is directed outwards. By neglecting the direct contribution of the external conducting surfaces of the PCB, the electric field can be calculated from the vector potential F (e.g. in [12]):

$$
\begin{aligned}
& \mathbf{F}=\epsilon \int_{S} \frac{\mathbf{M}_{\mathbf{s}} e^{-j k r}}{4 \pi r} d S^{\prime} \\
& \mathbf{E}=-\frac{1}{\epsilon} \nabla \times \mathbf{F}=-\int_{S} \mathbf{M}_{\mathbf{s}} \times \nabla \frac{e^{-j k r}}{4 \pi r} d S^{\prime}
\end{aligned}
$$

Assuming far field conditions, Eq. (2) can be further simplified according to [12], but in the present calculations the general equation has been used.

It can be observed that the general procedure of this type of calculation of the radiated field is well known in the literature, for example in [13], [14] and [2].

\subsection{Radiation from the Top Plane}

The second excitation mechanism of the radiation is directly the common mode current $I_{c}$. The current $I_{1}$ coming out from the PCB in the node $N_{g b}$ and the pin current $I_{2}$ inject a current $I_{c}$ into the ground plane. Since the two pins are very close to each other, the differential current has a negligible effect to the radiation at the considered frequencies, and the two pins can be considered as a single one carrying a current $I_{c}$, as far as the radiation is concerned.

Due to the small dimensions of the module, the exact current distribution on the module is probably not relevant for the radiation, and the simplified model shown in Fig. 6 and discussed in Sect. 3 can be used. Therefore, the same MoM calculation used for the calculation of the complex parasitic impedance has been used for the calculation of the radiation. The calculated field has been later normalized to the current of the voltage generator.

\subsection{Combination of the Radiation Mechanisms}

With the two techniques discussed in the previous sections two different complex transfer functions between a unit current and the electric field in one spatial position can be calculated. Each of these transfer function has been later multiplied by one complex current ratio between either the current $I_{1}$ in the PCB via or the common mode current $I_{c}$, respectively, and the IC current $I_{I C}$. The complex current ratios have been calculated in frequency domain with HSPICE, by using the equivalent circuit shown in Fig. 5 with a unitary IC current.

The two resulting complex products have been added, and thus the ratio between the electric field in one angular position and the IC current has been obtained. The process has been repeated for each angular position, and the maximum value of the $z$-component of the field has been calculated. It can be noted that all the calculations up to this stage have been conducted with complex quantities, because the two radiation mechanisms add with different phase shifts at each position, and are excited by different currents.

Finally, the transfer ratio between the maximum field value and the IC current has been multiplied by the IC current of the LECCS model. The overall process is schematically shown in Fig. 9. The three processes with the thick dotted line can be calculated independently from each other with a unit current source.

It is interesting to compare the contribution to the radiation of each radiation mechanism. To this purpose, the maximum transfer ratio in all angular position between the electric field and its respective excitation current has been calculated and plotted in Fig. 10. Since the radiation from the top plane is normalized to the current $I_{c}$, and the radi-

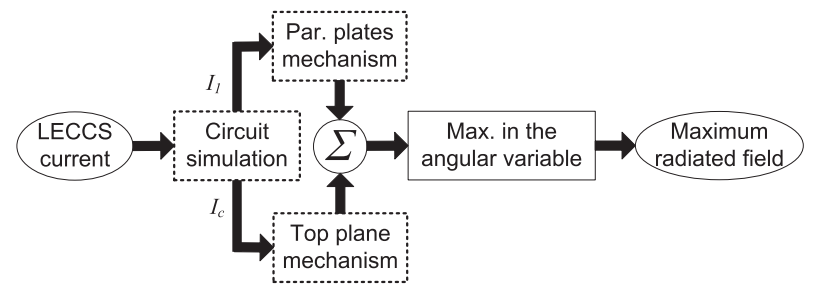

Fig. 9 Calculation of the maximum field

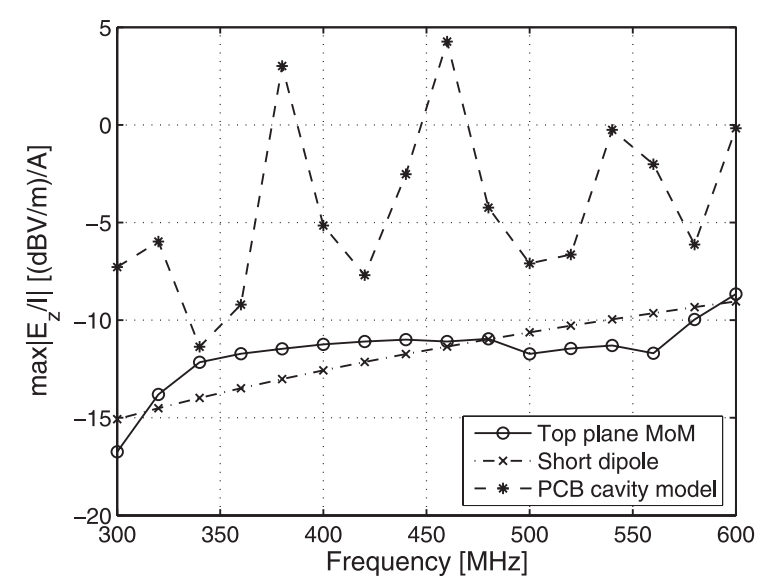

Fig. 10 Maximum radiated field per unit current $\left(I_{c}\right.$ and $\left.I_{1}\right)$. 
ation from the PCB power/ground planes is normalized to the via current $I_{1}$, it is not possible at this stage to say which mechanism is dominant. However, it can be observed that the radiation mechanism due to the power/ground planes is strongly affected by the PCB resonances, and it is much more effective than the mechanism due to the top plane at the peak values, whereas they are almost comparable at the dips.

It is also interesting to observe in the same figure, that the radiation from the top plane in the considered positions and polarization can be very simply approximated by the radiation of a short dipole of length equal to two times the distance between the two planes $(2.8 \mathrm{~mm})$. In other words, the top plane radiation seems to be mainly due to the pin common mode current and to its image due to the PCB, even without considering the floor of the semi-anechoic chamber. However, this drastic simplification of the model needs further evidence before extending its validity to other positions and polarizations. It would be also interesting to verify whether, by additionally considering also the loop current due to the pin common mode current and the return through the capacitance, the simplified model can be improved.

\section{Simulation Results}

\subsection{Common Mode and via Current}

The current ratios between the current into the pins, $I_{1}$ or $I_{2}$, or the common mode current, $I_{c}=I_{1}+I_{2}$, and the IC current obtained from the circuit in Fig. 5 for the cases with and without the impedance $Z_{c m}$ are shown in Fig. 11. When the impedance $Z_{c m}$ is not considered, the pin currents have the same magnitude $\left(I_{1}=-I_{2}\right)$ and the common mode current is zero.

It can be observed that when all the inductance is on the power line $\left(L_{g d i}=0 \mathrm{nH}, L_{v d i}=33 \mathrm{nH}\right)$, the impedance $Z_{c m}$ has almost no effect on the via current $I_{1}$. However, the common mode current $I_{c}$ increases with the frequency, and it becomes comparable and even larger than the via current $I_{1}$ at the higher frequencies. This is due to a resonance in the ground loop within the gray dotted line in Fig. 5, which is formed by the module ground plane, the ground interconnection, and by the PCB ground plane. In fact, as it is shown in Fig. 12, the relatively low ground loop impedance between the PCB ground plane node, $N_{g b}$, and the module ground plane node, $N_{g m}$, increases with frequency.

The presence of some resonances with relatively large magnitude is more evident in Fig. 12 below $450 \mathrm{MHz}$ for the cases when an inductor is present on the ground line. This has also a strong effect on the via current $I_{1}$ in Fig. 11, which becomes much smaller than the common mode $I_{c}$ around the resonance frequencies. The position of the resonance frequency is slightly different in the two figures, due to the presence of the mutual inductances, which have not been considered in the calculation of the ground loop impedance.

With reference to Fig. 5, in its way back to the current source, the via current $I_{1}$, flowing out from the power line
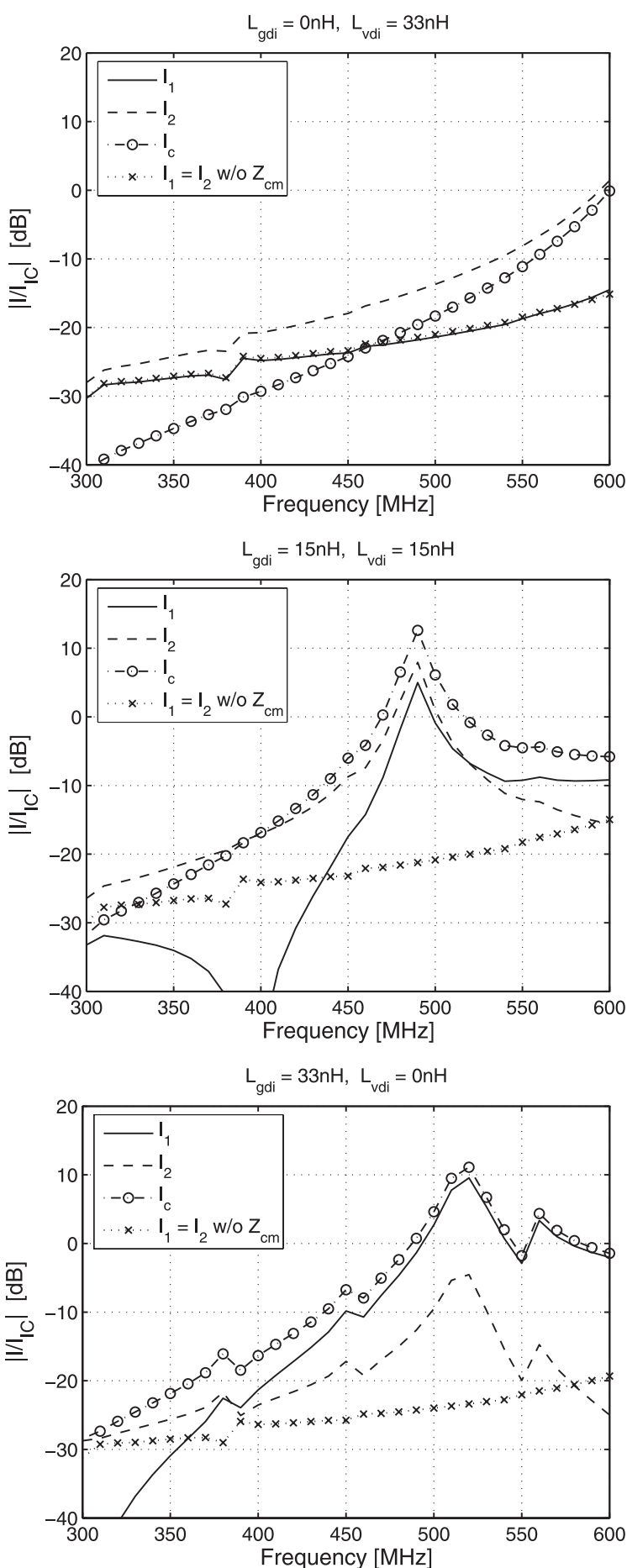

Fig. 11 Current transfer ratio with and without (w/o) $Z_{c m}$.

of the module, at the resonance frequency encounters a very large impedance at the node $N_{g b}$, due to the resonance of the ground plane loop. Therefore, instead of flowing in the power line, the current is diverted into the capacitance $C_{p g}$ on the module. At the same time, a relatively large common mode current $I_{c}$ flows in the ground loop.

It can be also observed that when an inductor is on the ground line, above $450 \mathrm{MHz}$ both the via and the common 


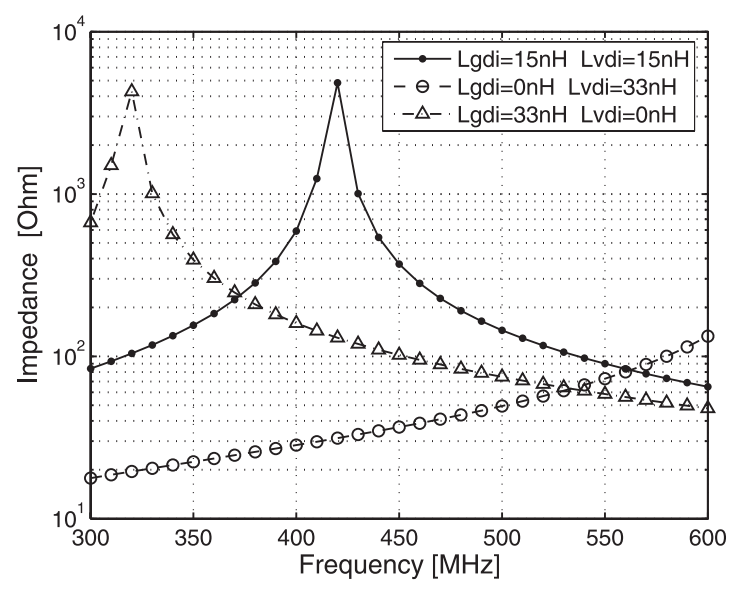

Fig. 12 Ground loop impedance [6].

mode current become relatively large. This is probably the reason for the increase in the measured radiated field observable in Fig. 2 at these frequencies, which is one of the rationale for this study. When the parasitic impedance $Z_{c m}$ is not considered, the via current $I_{1}$ is much smaller, as shown in Fig. 11, leading to an underestimation of the radiated field.

Other experiments have shown that the position of the peaks with large current are dependent on the value of the parasitic impedance $Z_{c m}$, and therefore of its parasitic capacitance. When this resonance is combined with the PCB ground plane resonances in Fig. 10 in the calculation of the radiation, a small variation of this impedance can have a considerable effect on the estimated radiated field.

\subsection{Radiated Field}

\subsubsection{Contributes to the Radiation}

The currents $I_{1}$ and $I_{c}$ are used to calculate the radiation as explained in Sect.4. The total calculated field is shown in Fig. 13 together with the contributes of each radiation mechanism and with the measured field. In the figure, the same marks as in Fig. 2 and in the following figures have been used for consistency.

It can be observed that the increase in the radiation above $450 \mathrm{MHz}$ observed in the measurements when there is an inductor in the ground line is rebuilt in the simulations as expected according to the relatively large currents in Fig. 11. At some frequencies the radiation is overestimated. This can be due to the incertitude in the distance between module and PCB, which can directly affect the value of the parasitic capacitance and the associated parasitic inductance, as well as the pin length, which affects their inductance and the direct contribution to the radiation from the top plane.

It can be also observed, that at the frequencies corresponding to the ground loop resonances, the dominant radiation mechanism is that due to the common mode current $I_{c}$ flowing on the pins and on the top plane. At all the other frequencies the parallel plates mechanism is sufficient for a rough estimation of the radiation, even if the top plane
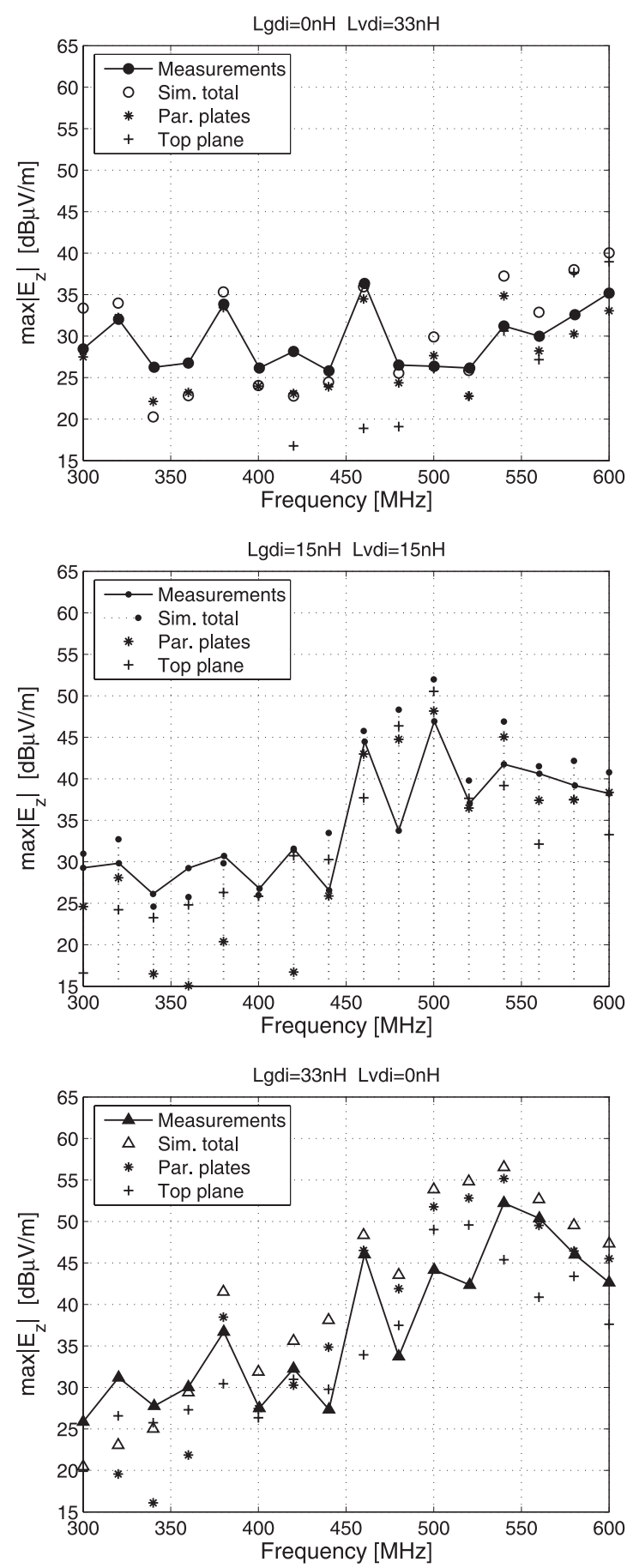

Fig. 13 Field with different radiation mechanisms.

mechanism is not negligible.

At few frequencies, the total radiated field is smaller than the sum of the radiated fields of each radiation mechanism. This is due to the fact that in the calculation scheme described in Fig. 9, the contributes of the two considered radiation mechanisms are combined as complex quantities, and in some cases they partially counterbalance each other. 


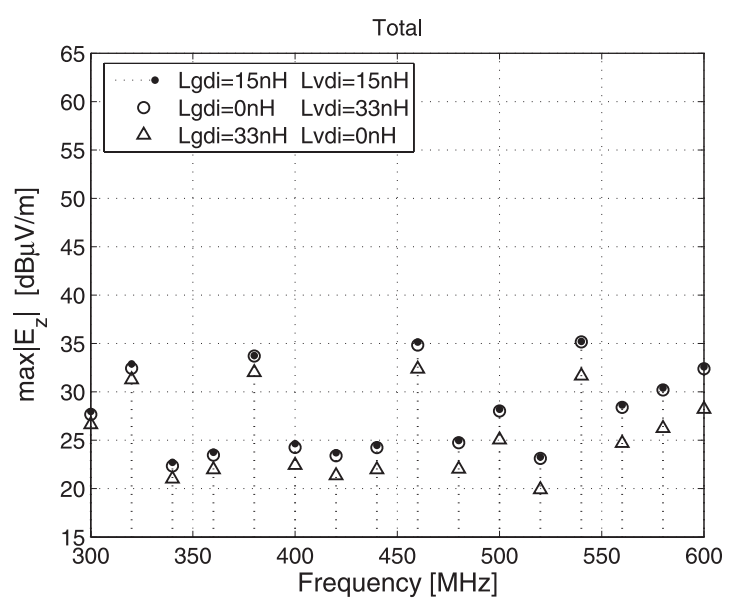

Fig. 14 Estimated electric field without $Z_{c m}$.

\subsubsection{Total Field with and without Capacitance}

The simplest model of the DUT can be obtained by completely neglecting the interaction between module and PCB, as it is sometimes done in package modeling. In terms of the equivalent circuit of Fig. 5, this corresponds to neglecting the parasitic impedance $Z_{c m}$. The resulting maximum radiated electric field at $3 \mathrm{~m}$ distance, considering also the reflection from the floor of the semi-anechoic room, is shown in Fig. 14.

By comparing the calculated values in Fig. 14 with the measurement results in Fig. 2, it can be observed that except for the case without inductance on the ground line $\left(L_{g d i}\right.$ $=0 \mathrm{nH}, L_{v d i}=33 \mathrm{nH}$ ), the differences are extremely large, even larger than $20 \mathrm{~dB}$ at some frequencies above $450 \mathrm{MHz}$. Furthermore, the model cannot predict the effect of the location of the discrete inductor on the power or ground lines, because the differences between the three sets of points are too small. The small reduction in radiation when all the inductance is concentrated on the ground line $\left(L_{\text {gdi }}=33 \mathrm{nH}\right.$, $L_{v d i}=0 \mathrm{nH}$ ) is mainly due to the parasitic capacitance $C_{1}$ between the module ground line and the module ground plane, which partly bypasses the inductance $L_{g d i}$.

When the parasitic impedance $Z_{c m}$ is added, there is a drastic improvement toward the measurement results, as shown in Fig. 15, where the results of Fig. 13 are summarized. It can be observed that a large difference of the field exists when the location of the inductor is changed, and that at the higher frequencies the field values are considerably larger. Even if some inaccuracies remain, which probably are related to the incertitude on the distance between the ground planes, the results clearly show that the considered parasitic capacitance is a key parameter for the estimation of the radiation, and for the dependency of the radiation on the location of the decoupling inductors.

This can be explained by the fact that the impedance $Z_{c m}$ represents an alternative return path for the current that flows out the power/ground plane impedance $Z_{p c b}$ at node $N_{g b}$, and therefore it neutralizes the effect of the inductor

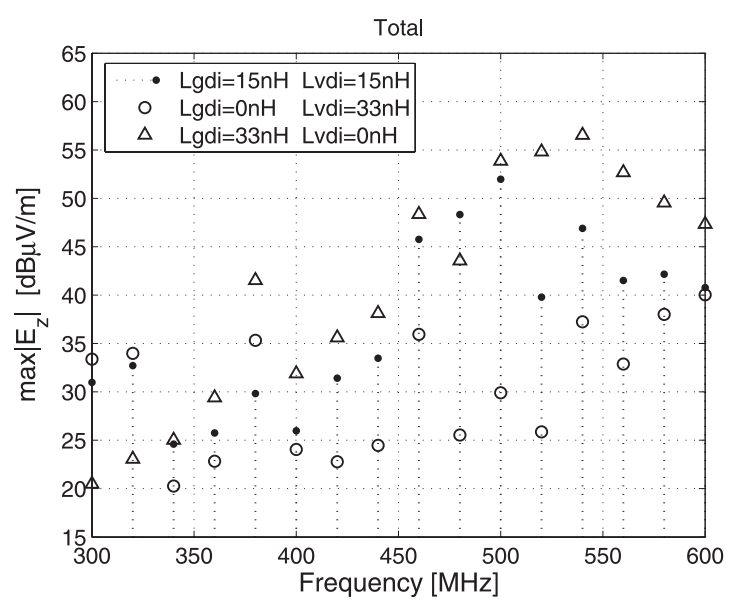

Fig. 15 Estimated electric field with $Z_{c m}$ [7].

on the ground line $L_{g d i}$. A second effect of the parasitic capacitance is to create the ground plane loop indicated in Fig. 5, which can resonate as already discussed.

\section{Conclusions}

From this study it should be clear that the parasitic capacitance between package and PCB has a very strong effect on the common mode current flowing out from the package, and it represents its main return path. A portion of this common mode current can enter the power/ground planes of the PCB, and when it is of the same order of the differential type current, it can cause large electromagnetic radiation and potentially other types of disturbances in the PCB. Furthermore, combined with the current on the PCB ground plane, the common mode current represents an important direct contribution to the radiation at some frequencies.

The usage of decoupling inductors on the ground line of the package can bring to undesirable effects. In order to model these effects, it is necessary to consider the parasitic capacitance between package and PCB.

When a decoupling inductor is put on the power line, the parasitic capacitance does not affect the via current in the power/ground planes, and the radiation is drastically reduced. However, some common mode current can still flow on the ground loop between module and PCB ground planes, and this can affect the radiation at higher frequencies by means of its direct contribution to the radiation in combination with the PCB ground plane. In order to model this effect, it is again necessary to consider the parasitic capacitance between package and PCB in the equivalent circuit.

It can be observed that the better performance of the power plane inductor in the considered example is also due to the particular DUT configuration, where the module and PCB ground planes were facing each other. It is arguable that in the dual situation with two power planes facing each other, the inductor in the power line combined with parasitic capacitance between module and PCB power planes may cause problems similar to those shown in this paper for the ground inductor. However, this has not been verified yet and 
it cannot be taken for granted in practice, because even in the dual situation usually the impedance of the ground line is kept low by design.

\section{Acknowledgments}

The presented work has been carried out within a project supported by STARC (www.starc.or.jp).

\section{References}

[1] H. Osaka, D. Tanaka, O. Wada, and R. Koga, "Linear equivalent circuit and current source model with I/O (LECCS-I/O) modeling of IC power current for EMI simulation," J. Japan Institute of Electronics Packaging, vol.7, no.6, pp.517-524, Sept. 2004.

[2] M. Nishida, Y. Toyota, K. Iokibe, R. Koga, and O. Wada, "Fast calculation of radiated emissions from arbitrarily shaped PCB with IC/LSI," Proc. 4th Int. Symp. on Electromagnetic Compatibility, Qingdao, China, pp.23-25, Oct. 2007.

[3] Z.L. Wang, O. Wada, Y. Toyota, and R. Koga, "Convergence acceleration and accuracy improvement in power bus impedance calculation with a fast algorithm using cavity modes," IEEE Trans. Electromagn. Compat., vol.47, no.1, pp.2-9, Feb. 2005.

[4] O. Wada, K. Takayama, R. Koga, Z.L. Wang, and Y. Fukumoto, "Effect of power supply decoupling for the reduction of EMI in power supply system of multilayer PCB," 3rd Technical Meeting of the JIEP Technical Group on Electromagnetic Characteristics, Feb. 2002.

[5] U. Paoletti, T. Hisakado, and O. Wada, "Effect of package parasitics on conducted and radiated emission with mixed mode analysis," Proc. Asia-Pacific EMC Week, Singapore, May 2008.

[6] U. Paoletti, T. Hisakado, and O. Wada, "Effect of package commonmode current on PCB power bus noise and radiation," Proc. International Conference on Electronics Packaging (ICEP 2008), pp.150155, Tokyo, June 2008.

[7] U. Paoletti, T. Hisakado, and O. Wada, "Importance and limitations of modeling parasitic capacitance between package and PCB for power bus noise and radiation," Proc. 3rd Pan-Pacific EMC Joint Meeting (PPEMC'08), Tokyo, pp.5-6, May 2008.

[8] U. Paoletti and O. Wada, "De-embedding technique for the extraction of parasitic and stray capacitances from 1-port measurements," IEICE Trans. Commun., vol.E90-B, no.6, pp.1298-1304, June 2007.

[9] K. Nabors and J. White, "Multipole-accelerated capacitance extraction algorithms for 3-D structures with multiple dielectrics," IEEE Trans. Circuits Syst. I, Fundam. Theory Appl., vol.39, no.11, pp.946-954, Nov. 1992.

[10] M. Kamon, M.J. Tsuk, and J. White, "A multipole accelerated 3-D inductance extraction program," Proc. 30th international conference on Design automation, pp.678-683, Dallas, Texas, 1993.

[11] J.D. Morsey, B.J. Rubin, L. Jiang, L. Shan, L.B. Eisenberg, D. Becker, and M. Arseneault, "The use of fast integral equations solvers for practical package and interconnect analysis," Proc. Elec. Performance of Electronic Packaging, pp.335-338, Oct. 2006.

[12] S. Ramo, J.R. Whinnery, and T.V. Duzer, Fields and Waves in Communication Electronics, third ed., J. Wiley \& Sons, New York, 1993.

[13] M. Leone, T. Fischer, and M. Albach, "Investigation of the radiation from parallel-plane power-bus structures on printed circuit boards," Electrical Engineering (Archiv für Elektrotechnik), vol.85, no.2, pp.113-118, 2003.

[14] M. Leone, "The radiation of a rectangular power-bus structure at multiple cavity-mode resonances," IEEE Trans. Electromagn. Compat., vol.45, no.3, pp.486-492, Aug. 2003.

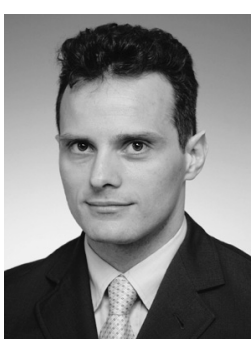

Umberto Paoletti received the degree in Electronic Engineering from the University of Ancona, Italy, in 1998, and the Dr.E. degree from the University of Hanover, Germany in 2005. Between 1999 and 2000 he was officer of the Technical Corp of the Italian Army. Between 2000 and 2005 he was working for the University of Paderborn, Germany, at the Fraunhofer Institute for Reliability and Microintegration in Berlin, Germany. Since 2005 he has been working in the Department of Electrical Engineering at the Graduate School of Engineering of Kyoto University, Kyoto Daigaku Katsura, Nishikyo-ku, 615-8510 Japan. Dr. Paoletti is a member of IEEE.

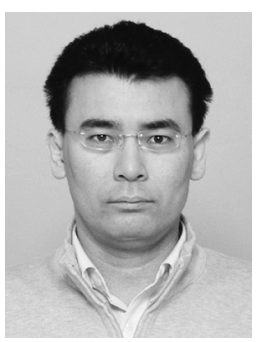

Takashi Hisakado received the B.E. and M.E. degrees in Electrical Engineering II from Kyoto University, in 1993 and 1995, respectively. He received the Dr. degree in Electrical Engineering from Kyoto University, in 1997. $\mathrm{He}$ is currently Associate Professor of Department of Electrical Engineering at Kyoto University. His research interests are nonlinear circuit, power circuit, EMC design, FPGA implementation, signal processing and so forth. $\mathrm{He}$ is a member of IEEJ, and IEEE.

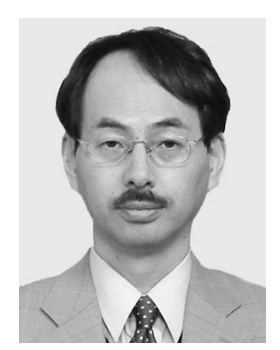

Osami Wada was born in 1957. He received the B.E., M.E. and Dr.E. degrees in electronics from Kyoto University, Japan, in 1981, 1983 and 1987 respectively. Between 1988 and 2005 he was working at the Faculty of Engineering of Okayama University, Japan. Since 2005 he is Full Professor at the Department of Electrical Engineering at the Graduate School of Engineering of Kyoto University, Kyoto Daigaku Katsura, Nishikyo-ku, 615-8510 Japan. Prof. Wada is a member of IEE of Japan, the Japan Institute of Electronics Packaging, and IEEE. 